\title{
Renormalized dissipation in the nonconservatively forced Burgers equation
}

\author{
John A. Krommes \\ Princeton University, P.O. Box 451, Princeton, New Jersey 08543-0451, USA
}

(June 24, 1999)

\begin{abstract}
A previous calculation [P. H. Diamond and T.-S. Hahm, Phys. Plasmas 2, 3640 (1995)] of the renormalized dissipation in the nonconservatively forced one-dimensional Burgers equation, which encountered a catastrophic long-wavelength divergence $\sim k_{\min }^{-3}$, is reconsidered. In the absence of velocity shear, analysis of the eddy-damped quasi-normal Markovian closure predicts only a benign logarithmic dependence on $k_{\mathrm{min}}$. The original divergence is traced to an inconsistent resonancebroadening type of diffusive approximation, which fails in the present problem. Ballistic scaling of renormalized pulses is retained, but such scaling does not, by itself, imply a paradigm of selforganized criticality. An improved scaling formula for a model with velocity shear is also given.
\end{abstract}

PACS: 52.35.Ra, 05.60.+w

In a famous calculation of the "large-distance and longtime properties of a randomly stirred fluid," Forster, Nelson, and Stephen ${ }^{1}$ (FNS) analyzed the consequences of various forcing scenarios for the Navier-Stokes equation and, to some extent, Burgers equation. They considered both a conservative forcing (Model A) and a nonconservative one (Model B), ${ }^{2}$ and predicted nontrivial properties for the lowfrequency, small-wave-number limits of the two-point correlation and response functions. They did not explicitly consider Model B for Burgers equation; however, that was later studied in considerable detail by Hwa and $\operatorname{Kardar}^{3}$ (HK). Recently Diamond and $\mathrm{Hahm}^{4}$ (DH) attempted to use the Burgers Model B in support of a paradigm of self-organized criticality $^{5,6}$ (SOC) for plasma transport. In the course of their discussion, they performed a calculation of the renormalized dissipation coefficient $\eta_{k}$ that describes the mean propagation of small-amplitude pulses with Fourier components $k$. In the absence of macroscopic velocity shear $V^{\prime}$, they invoked a wave-number scaling for the turbulent dissipation $\left(\eta_{k} \sim k^{2}\right)$ that disagreed with that predicted by HK $\left(\eta_{k} \sim|k|\right)$. As a consequence, they encountered a catastrophic long-wavelength divergence $\sim \int_{k_{\min }} d q / q^{4}$, where $k_{\text {min }}$ is a minimum wave-number cutoff. Their result for $V^{\prime} \neq 0$ also exhibited pathologies. In the present work, I reconsider the calculations. For $V^{\prime}=0$, I find only a benign logarithmic divergence and a wave-number scaling in agreement with HK. I remark that such scaling, although anomalous, does not by itself point to an SOC paradigm. Finally, I deduce a more satisfactory scaling formula for $V^{\prime} \neq 0$.

The 1-D forced Burgers equation is

$$
\partial_{t} u(x, t)+u \partial_{x} u-\mu \partial_{x}^{2} u=f^{(\mathrm{ext})}(x, t),
$$

where $\mu$ is a small parameter and in Model B it is assumed that the random noise $f^{(\text {ext })}$, taken to be a centered Gaussian with variance $F^{(\mathrm{ext})}$, is nonconservative:

$$
\begin{aligned}
F^{(\mathrm{ext})}(\rho, \tau) & \doteq\left\langle f^{(\mathrm{ext})}(x+\rho, t+\tau) f^{(\mathrm{ext})}(x, t)\right\rangle \\
& =F_{0}^{(\mathrm{ext})} \delta(\rho) \delta(\tau)
\end{aligned}
$$

$$
\begin{aligned}
F_{k, \omega}^{(\mathrm{ext})} & \doteq \int_{-\infty}^{\infty} d \rho e^{-i k \rho} \int_{-\infty}^{\infty} d \tau e^{i \omega \tau} F^{(\mathrm{ext})}(\rho, \tau) \\
& =F_{0}^{(\mathrm{ext})}=\mathrm{const} .
\end{aligned}
$$

[Model A of FNS corresponds to conservative forcing with $F_{k, \omega}^{(\text {ext })}=2 k^{2} D$.] Equation (1) can be represented in the standard mode-coupling form

$$
\left(\partial_{t}+k^{2} \mu\right) u_{k}=\frac{1}{2} \sum_{\Delta} M_{k p q} u_{p}^{*} u_{q}^{*} \quad\left(M_{k p q}=-i k\right),
$$

where $\sum_{\Delta}$ means the sum over all triangles such that $k+p+q=0$.

The statistical theory of equations such as (4) is well developed, ${ }^{7-9}$ with the Eulerian direct-interaction approximation (DIA) ${ }^{10}$ being unique from several points of view. ${ }^{11}$ DH asserted that they were solving the DIA, but in fact they were not. The DIA is a particular, rather complicated set of cubically nonlinear integro-differential equations involving time-history integrations, and DH made no attempt to deal with those. Rather, they quite reasonably attempted to analyze the consequences of the selfconsistency embodied not only in the DIA but also in more workable closures such as the eddy-damped quasi-normal Markovian (EDQNM) approximation ${ }^{12}$ and even Dupree's early resonance-broadening theory (RBT). ${ }^{13}$ Their analysis is closest in spirit to the RBT. Unfortunately, one of the fundamental postulates of RBT - that turbulent dissipation can be approximated at small $k$ 's by a term $k^{2} D$ that renormalizes the classical viscous damping - turns out to be violated in the present problem, as was shown quite generally by HK and will be demonstrated more specifically here.

The DIA-based EDQNM ${ }^{14,15}$ possesses the same selfconsistency, mode-coupling, and scaling properties as the DIA itself; it is adequate for a rough analysis. ${ }^{16}$ The approximation can be defined in terms of the nonlinear Langevin equation ${ }^{17}$

$(\doteq$ means definition $)$, or 


$$
\left(\partial_{t}+k^{2} \mu+\widehat{\eta}_{k}\right) u_{k}=f_{k}^{(\mathrm{int})}+f_{k}^{(\mathrm{ext})},
$$

where $\widehat{\eta}_{k}$ represents turbulent damping and $f_{k}^{(\mathrm{int})}$ is internal nonlinear noise [with variance $F^{(\mathrm{int})}(\tau)$ ] arising from the mode coupling. In terms of the (equal-time) fluctuation spectrum $C_{k} \doteq\left\langle\left|\delta u_{k}\right|^{2}\right\rangle$ and the triad interaction time $\theta_{k p q}$, one has

$$
\widehat{\eta}_{k}=-\sum_{\Delta} M_{k p q} M_{p q k}^{*} \theta_{k p q}^{*} C_{q}
$$

and $F_{k}^{(\mathrm{int})}(\tau)=F_{k}^{(\mathrm{int})} \delta(\tau)$, with

$$
F_{k}^{(\mathrm{int})} \doteq \frac{1}{2} \sum_{\Delta}\left|M_{k p q}\right|^{2} \operatorname{Re} \theta_{k p q} C_{p} C_{q} .
$$

In general, $\theta_{k p q}$ evolves according to

$$
\partial_{t} \theta_{k p q}+\left(\eta_{k}+\eta_{p}+\eta_{q}\right) \theta_{k p q}=1,
$$

where $\eta_{k} \doteq k^{2} \mu+\widehat{\eta}_{k}$ is the total dissipation. For present purposes, one may use the time-asymptotic result $\lim _{t \rightarrow \infty} \theta_{k p q}=\left(\eta_{k}+\eta_{p}+\eta_{q}\right)^{-1}$ in the calculations of $\widehat{\eta}_{k}$ and $F_{k}^{\text {(int) }}$.

According to FNS, the exact infinitesimal response function $R_{k, \omega}$ should at small $k$ and $\omega$ obey the scaling

$$
R_{k, \omega}^{-1} \sim|k|^{-z} g_{R}\left(\omega /|k|^{z}\right)
$$

for some undetermined scaling function $g_{R}$ and scaling exponent $z$. If their results for the Model-B Navier-Stokes problem are extended to spatial dimensionality $d=1$, they imply ${ }^{18} z=1$, in agreement with the explicit calculations of HK for the 1-D Burgers equation. Now in steady state the response function for the dynamics described by Eq. (5) is $R_{k, \omega}^{-1}=-i\left(\omega+i \eta_{k}\right)$. Thus, the result $z=1$ implies $\eta_{k} \propto|k|$.

The turbulent damping $\widehat{\eta}_{k}$ must be self-consistently calculated from Eq. (6), given the steady-state spectrum $C_{q}$. That is determined from the time-asymptotic solution of the spectral balance equation

$$
\partial_{t} C_{k}+2 \operatorname{Re} \eta_{k} C_{k}=2\left(F_{k}^{(\text {int })}+F_{k}^{(\text {ext })}\right),
$$

or

$$
C_{k}(t=\infty)=\left(F_{k}^{(\mathrm{int})}+F_{k}^{(\mathrm{ext})}\right) / \operatorname{Re} \eta_{k} .
$$

Subsequently, I shall write $C_{k} \equiv C_{k}(t=\infty)$. According to Eqs. (6) and (7), Eq. (11) is a self-consistent equation for $C_{k}$.

With the scaling $\eta_{k} \sim|k|$, the classical dissipation can be neglected for small $k\left(\eta_{k} \rightarrow \widehat{\eta}_{k}\right)$, whereupon Eq. (11) suggests that $C_{k} \sim|k|^{-1}$. If so, then the internal noise scales as

$$
F_{k}^{(\mathrm{int})} \sim k^{2} \int d q \frac{1}{|k|+|k+q|+|q|} \frac{1}{|k+q|} \frac{1}{|q|},
$$

or $F_{k}^{(\text {int })} \sim$ const. Thus the internal noise renormalizes ${ }^{19}$ $F^{\text {(ext) }}$, giving rise to a total forcing $F_{k}=F_{k}^{(\text {int })}+F_{k}^{\text {(ext) }}$ that approaches a constant $F_{0}$ as $k \rightarrow k_{\min }$. I shall not attempt to calculate the details of $F_{k}$ in the present paper, since I wish to focus on the scaling properties of $\eta_{k}$. Equation (6) becomes

$$
\widehat{\eta}_{k}=\int \frac{d q}{2 \pi} k(k+q)\left(\frac{1}{\widehat{\eta}_{k}+\widehat{\eta}_{k+q}+\widehat{\eta}_{q}}\right)\left(\frac{F_{q}}{\widehat{\eta}_{q}}\right) .
$$

(Note that the existence of $k_{\min }$ implies additional constraints, not written here, on the integration domain that ensure that the triangle relation $k+p+q=0$ is satisfied.) Because of the last factor of $\widehat{\eta}_{q}^{-1}$, the $q$ integral in Eq. (13) is dominated by the small $q$ 's, so again the classical dissipation can be neglected. To determine the self-consistent $k$ scaling implied by Eq. (13), one may scale $q$ to $k$. One readily finds that

$$
\widehat{\eta}_{k}=|k| \bar{V}(\widehat{k}),
$$

where a dependence of $\bar{V}$ on $\widehat{k} \doteq|k| / k_{\text {min }}$ is allowed. To the extent that dependence on $\widehat{k}$ is ignored, Eq. (14) agrees with Eq. (9) with the HK result $z=1$.

The precise functional form of $\bar{V}$ cannot be obtained analytically; it is approximately

$$
\bar{V}(\widehat{k})=\left(F_{0} / 2 \pi\right)^{1 / 3} \psi(\widehat{k}), \quad \psi(\widehat{k}) \approx \ln \widehat{k}+\frac{1}{2} \widehat{k}^{-1} .
$$

This $\widehat{k}$ dependence requires some discussion, since for $k \sim$ $k_{\min }$ one has $\widehat{\eta}_{k} \sim k_{\min }$, which appears to violate the basic linear scaling with $|k|$. The resolution is that in the renormalization-group (RNG) calculations of FNS and HK it was assumed that $k_{\min } \rightarrow 0$ (in which case $k_{\min }$ cannot enter into any dimensional result). That limit also implies $|k| / k_{\min } \equiv \widehat{k} \gg 1$. The slowly varying logarithmic correction that one obtains in that regime cannot be obtained from simple RNG calculations.

In contrast with the above self-consistent EDQNM calculation, DH postulated (in the spirit of RBT) that $\widehat{\eta}_{k} \sim$ $k^{2} D$, leading them to encounter a divergence $\int_{k_{\min }} d q / q^{4}$ (it would be $\int_{k_{\min }} d q / q^{2}$ in an analysis parallel to the one above) and to misleading attempts to interpret the strong dependence of $\widehat{\eta}_{k}$ on the cutoff $k_{\text {min }}$. However, $\widehat{\eta}_{k} \sim k^{2} D$ is not a self-consistent solution for this particular forcing scenario and 1-D model.

Now consider the generalization to $d=2$. By definition, the model studied by DH possesses an advective nonlinearity acting only in the $x$ direction. I initially ignore velocity shear. One must therefore analyze the 2-D integral equation

$\widehat{\eta}_{\boldsymbol{k}}=\int \frac{d \boldsymbol{q}}{(2 \pi)^{2}} k_{x}\left(k_{x}+q_{x}\right)\left(\frac{1}{\widehat{\eta}_{\boldsymbol{k}}+\widehat{\eta}_{\boldsymbol{k}+\boldsymbol{q}}+\widehat{\eta}_{\boldsymbol{q}}}\right)\left(\frac{\mathcal{F}_{\boldsymbol{q}}}{\widehat{\widehat{\eta}}_{\boldsymbol{q}}}\right)$

where $\mathcal{F}_{\boldsymbol{q}}$ is the 2-D generalization of $F_{q}$. If ones ignores classical dissipation, the only $q_{y}$ dependence comes 
from $\mathcal{F}_{\boldsymbol{q}}$. In order that the $q_{y}$ integral be well defined and that the model reduces properly to $d=1$, one must assume that the forcing is integrable, $(2 \pi)^{-1} \int d q_{y} \mathcal{F}_{\boldsymbol{q}}=F_{0}$; for later estimates, I assume that $\mathcal{F}\left(q_{y}\right)$ falls off rapidly for $q_{y}$ greater than some characteristic wave number $k_{*}$. It can then be seen that one recovers Eq. (14) with $k \rightarrow k_{x}$. (Had the advective nonlinearity been more physically generalized to $d=2$, different results would have been obtained.)

Next, I follow DH and consider the consequences of a shearing term $V^{\prime} x \partial_{y} u$ added to the left-hand side of the 2 -D version of Eq. (1). That term is directly analogous to the $k_{\|}(x) v_{\|}=k_{y} x L_{\mathrm{s}}^{-1} v_{\|}$streaming term encountered in the theory of particle motion in sheared magnetic fields ( $L_{\mathrm{s}}$ being the magnetic shear length), and in that context was analyzed extensively in Ref. 20. The fundamental result, which dates back to earlier work by Dupree ${ }^{21}$ on the RBT of the 1-D Vlasov equation (there the roles of $x$ and $v$ are interchanged), is that in the presence of an assumed $x$-space diffusion $k_{x}^{2} D$ the competition between shear and diffusion leads to a reduced autocorrelation time $\tau_{\mathrm{c}}\left(k_{y}\right) \sim\left(k_{y}^{2} V^{\prime 2} D\right)^{-1 / 3}$ comprising $2 / 3$ shear and $1 / 3$ diffusion [cf. Eq. (52) of Ref. 20]. This result was later used in Ref. 22 to discuss the effects of macroscopic velocity shear on the reduction of turbulent transport. ${ }^{23}$ For the Burgers model, DH made the important observation that when the shearing effect dominates, the wave-number scaling of $\eta_{\boldsymbol{k}}$ becomes $k_{x}^{2}$ rather than $\left|k_{x}\right|$, in accord with the diffusion hypothesis. That follows immediately from Eq. (16) if the denominator is assumed to be independent of $q_{x}$.

DH attempted to calculate the diffusion coefficient in the presence of shear. Their result is suspect for two reasons: (i) it diverges with an inverse power of a $k_{y, \min }$; (ii) it does not obey the same transport-reduction scaling that was found in Ref. 22. I shall sketch an improved estimate. (Although a complete numerical solution of the inhomogeneous and anistropic EDQNM closure is marginally feasible, it has not yet been done.) The basic idea is to perform the $\boldsymbol{q}$ integral in Eq. (16) in polar coordinates, integrating only over the region for which $\tau_{\mathrm{c}}^{-1}\left(q_{y}\right)$ dominates over the shear-free result (14). I specifically consider $D=$ $\lim _{k_{y} \rightarrow 0} \widehat{\eta}_{\boldsymbol{k}} / k_{x}^{2}$. The condition $\left(q_{y}^{2} V^{\prime 2} D\right)^{1 / 3}>\left|q_{x}\right| \bar{V}$ can be written in polar coordinates $\left(q_{x}=q \cos \theta, q_{y}=q \sin \theta\right)$ as

$$
q<f(\theta) V^{\prime 2} D / \bar{V}^{3}=f(\theta) \mathcal{S}^{3} k_{*}
$$

where $f(\theta) \doteq \sin ^{2} \theta /\left|\cos ^{3} \theta\right|$ and

$$
\mathcal{S} \doteq\left(\frac{V^{\prime}}{k_{*}^{2} D}\right)^{1 / 3}=\left(\frac{V^{\prime}}{\bar{V} k_{*}}\right)^{1 / 2}
$$

In writing Eqs. (17b) and (18b), I anticipated the final result (19a). $\mathcal{S}$ is an appropriately dimensionless measure of the shearing rate. ${ }^{24}$

With $\tau_{\mathrm{c}}^{-1}\left(q_{y}\right)$ replacing $\widehat{\eta}$ in the denominator of Eq. (16), and if any dependence of $\mathcal{F}$ on $q$ is temporarily ignored, the $q$ integral $\int q d q / q^{4 / 3} \propto q^{2 / 3}$ is convergent as $q \rightarrow 0$. However, the resulting integral $\bar{V}^{-2} \int d \theta \cos ^{-2} \theta=\bar{V}^{-2} \tan \theta$ is divergent at $\theta=\pi / 2,3 \pi / 2$ (i.e., at $q_{x}=0$ ). At those points the upper bound (17) diverges, contradicting the condition that $\mathcal{F}$ cuts off for $q_{y}>k_{*}$. From Eq. (17b), the condition $\left|q_{y, \max }\right|<k_{*}$ can be written as $\left|\tan ^{3} \theta\right|<\mathcal{S}^{-6} \beta^{-3}$, where one postulated that $D=\beta F_{0}^{1 / 3} / k_{*}$ and the dimensionless parameter $\beta$ is to be determined. This gives the scaling of the $\theta$ integral as $\bar{V}^{-2} \mathcal{S}^{-2} \beta^{-1}$. If one estimates $\mathcal{F} \sim F_{0} / k_{*}$ and notes that $\bar{V} \sim F_{0}^{1 / 3}$, self-consistency gives $\beta \sim \mathcal{S}^{-1}$ and the final results

$$
D \sim \mathcal{S}^{-1} F_{0}^{1 / 3} k_{*}^{-1}, \quad \tau_{\mathrm{c}}^{-1} \sim \mathcal{S}\left(k_{*} F_{0}^{1 / 3}\right)
$$

The validity condition $\tau_{\mathrm{c}}^{-1}\left(k_{y}\right)>\left|k_{x}\right| \bar{V}$, which defines the regime of strong shear, can then be written as $\mathcal{S}>\left|k_{x}\right| / k_{*}$, essentially $\mathcal{S} \gtrsim 1$ for an isotropic spectrum. One can also check that in this same regime one has $k_{x}^{2} D<\left|k_{x}\right| \bar{V}$. The $\mathcal{S}^{-1}$ reduction factor in Eq. (19a) is the same one that arises in the discussion ${ }^{23}$ of Ref. 22 (appearing there as the scaling with a reduced correlation time $\tau_{f}$ ). In making the connection, one must remember that the forcing scenarios differ between the two calculations.

The previous analysis corrects some technical errors in the calculations of DH and serves as an interesting example of the use of the EDQNM. Let us now turn to the scaling properties of the solution. The salient feature of the HK result is that for the 1-D Burgers equation with Model-B forcing the scaling (9) holds in the absence of shear. That corresponds to small pulses propagating, on the average, with the ballistic scaling $x \sim \bar{V} t$. DH attempted to draw a connection between this result and an SOC paradigm (for further discussion of such paradigms, see Ref. 25 and references therein) by reiterating a suggestion of HK that the 1-D Burgers equation is the appropriate continuum description of a sandpile model. Note, however, that dynamically diverse stochastic models can lead to the same scaling (9). For example, the random advection equation $^{26,27} \partial_{t} u+\widetilde{V} \partial_{x} u=0$, where $\widetilde{V}$ is a Gaussian random variable independent of space and time, has the exact response function $R_{k}(\tau)=H(\tau) \exp \left(-\frac{1}{2} k^{2} \bar{V}^{2} \tau^{2}\right)$ ( $H$ is the unit step function, $\bar{V} \doteq\left\langle\widetilde{V}^{2}\right\rangle^{1 / 2}$ ), which also scales according to Eq. (9). That model is devoid of the intermittent avalanche dynamics characteristic of the sandpile models of SOC. ${ }^{6}$

The additively and nonconservatively forced 1-D Burgers equation is not inevitably the proper description of long-wavelength phenomena in a real multidimensional system for reasons ranging from trivial to profound. For the mean fields, although it is true that statistical averages of multidimensional models lead to $1-\mathrm{D}$ equations that govern mean profile dynamics, such equations contain no random forcing by construction. For the fluctuations, realistic turbulent systems involve multiplicative rather than additive forcing. ${ }^{28}$ Although an advective nonlinear term can always be placed on the right-hand side of the dynamical equation and be called an additive forcing, that may not be useful because (i) the statistics of such a term are not arbitrary and specifically cannot be Gaussian (even for 
passive advection); (ii) such forcing would be conservative (Model A). But according to the results of FNS, Models A and $\mathrm{B}$ belong to different universality classes. Deeper issues relating to the effective long-wavelength noise for the Navier-Stokes equation were addressed in Ref. 29. Also, proper representation of realistic multidimensional plasma dynamics must take wave effects into account, which in general give a short ("microscopic") correlation time to the advecting velocity; ${ }^{28,25}$ that effect is not captured by the Burgers model.

This is not to say that the possibility that SOC may somehow be involved in plasma transport should not be investigated seriously. But it can be misleading to invoke SOC as the likely explanation for a variety of interesting long-wavelength, low-frequency self-similar scaling phenomena, ${ }^{30}$ because those can frequently be explained by simpler means. For example, in Ref. 25 it was shown by direct numerical simulation that a nontrivial and physically relevant stochastic model, possessing multiplicative forcing with short autocorrelation time but not obeying various central tenets of SOC such as submarginal dynamics, ${ }^{31}$ generates long-time tails on certain two-time correlations similar to those observed in experiment, ${ }^{32}$ as does the dynamically self-consistent Hasegawa-Wakatani model.

In conclusion, I have used the eddy-damped quasinormal Markovian statistical closure to calculate the longwavelength scaling of the turbulent dissipation $\widehat{\eta}_{k}$ for the nonconservatively forced Burgers models discussed in Ref. 4. In the absence of velocity shear, the result $\widehat{\eta}_{k} \propto|k|$ agrees with general results of Forster, Nelson, and Stephen ${ }^{1}$ and Hwa and Kardar; ${ }^{3}$ the postulate $\widehat{\eta}_{k} \approx k^{2} D$ fails for this problem. In the presence of shear, I proposed the new scaling results (19). The dependence on the shear parameter $\mathcal{S}$ [defined by Eq. (18b)] can be summarized by

$$
\widehat{\eta}_{\boldsymbol{k}} \sim \begin{cases}\left|k_{x}\right| \bar{V} & \left(\mathcal{S}<\left|k_{x}\right| / k_{*}\right) \\ \left(k_{x} / k_{*}\right)^{2} \mathcal{S}^{-1}\left(k_{*} \bar{V}\right) & \left(\mathcal{S}>\left|k_{x}\right| / k_{*}\right)\end{cases}
$$

where $\bar{V} \doteq F_{0}^{1 / 3}, F_{0}$ is the strength of the (renormalized) forcing, and $k_{*}$ is a characteristic wave number in the $y$ direction. This result is properly continuous across the boundary $\mathcal{S}=\left|k_{x}\right| / k_{*}=\mathcal{O}(1)$ and demonstrates the expected transport reduction for large shear with fixed forcing. For weak shear, the ballistic scaling $x \sim \bar{V} t$ obtains not just for this model, but for other, dynamically diverse stochastic systems as well. Accordingly, it cannot by itself be used to support a paradigm of self-organized criticality that involves sandpile-like dynamics.

\section{ACKNOWLEDGMENTS}

I am grateful to Maurizio Ottaviani for detailed discussions on Ref. 1 and many related topics in the theory of statistical renormalization and self-organized criticality. This research was supported by U.S. Department of Energy Contract No. DE-AC02-76-CHO-3073.
${ }^{1}$ D. Forster, D. R. Nelson, and M. J. Stephen, Phys. Rev. A 16, 732 (1977).

2 They also considered a Model C, involving band-limited forcing. They showed that the long-wavelength behavior of that model was the same as that of Model A.

3 T. Hwa and M. Kardar, Phys. Rev. A 45, 7002 (1992).

${ }^{4}$ P. H. Diamond and T.-S. Hahm, Phys. Plasmas 2, 3640 (1995).

${ }^{5}$ P. Bak, C. Tang, and K. Wiesenfeld, Phys. Rev. A 38, 364 (1988).

${ }^{6}$ H. J. Jensen, Self-Organized Criticality (Cambridge U. Press, Cambridge, UK, 1998).

${ }^{7}$ P. C. Martin, E. D. Siggia, and H. A. Rose, Phys. Rev. A 8, 423 (1973).

8 J. A. Krommes, in Handbook of Plasma Physics, edited by A. A. Galeev and R. N. Sudan (North-Holland, Amsterdam, 1984), Vol. 2, Chap. 5.5, p. 183.

9 J. A. Krommes, Phys. Rep. 283, 5 (1997).

${ }^{10}$ R. H. Kraichnan, J. Fluid Mech. 5, 497 (1959).

${ }^{11}$ R. H. Kraichnan, in New Perspectives in Turbulence, edited by L. Sirovich (Springer, New York, 1991), Chap. 1, p. 1.

12 S. A. Orszag, in Fluid Dynamics, edited by R. Balian and J.-L. Peube (Gordon and Breach, New York, 1977), p. 235.

13 T. H. Dupree, Phys. Fluids 10, 1049 (1967).

14 J. C. Bowman, Ph.D. thesis, Princeton University, 1992.

15 J. Bowman, J. A. Krommes, and M. Ottaviani, Phys. Fluids B 5, 3558 (1993).

16 The EDQNM fails in the presence of linear waves because statistical realizability breaks down. The Realizable Markovian Closure (Ref. 15), which corrects that deficiency, reduces by construction to the EDQNM for the present Burgers model (whose linear dynamics are purely dissipative).

17 C. E. Leith, J. Atm. Sci. 28, 145 (1971).

${ }^{18}$ For the Navier-Stokes Model B, FNS found [their Eq. (4.19)] $z=2-\frac{1}{3} \epsilon+\mathcal{O}\left(\epsilon^{2}\right)$ for $\epsilon=4-d$. Here $d=1$ and the $\mathcal{O}\left(\epsilon^{2}\right)$ term is treated as negligible in the absence of vertex renormalization.

19 This result differs from that of HK. They argued that because $F_{k}^{(\mathrm{int})} \propto\left|M_{k p q}\right|^{2}=k^{2}$, which vanishes as $k \rightarrow 0, F^{(\mathrm{int})}$ should also vanish in that limit. However, that logic is incomplete, since the $q$ integration can introduce the compensating factor $k^{-2}$ and that actually happens for $d=1$. As an example, consider the integral $\int d q(k+q)^{-2} q^{-1}=[k(k+q)]^{-1}-k^{-2} \ln ((k+q) / q)$.

20 J. A. Krommes, C. Oberman, and R. G. Kleva, J. Plasma Phys. 30, 11 (1983).

21 T. H. Dupree, Phys. Fluids 9, 1773 (1966).

${ }^{22}$ H. Biglari, P. H. Diamond, and P. W. Terry, Phys. Fluids B 2, 1 (1990)

23 Many of the detailed mathematical calculations in Ref. 22, which rely on a calculation of trajectory divergence to predict macroscopic spectral effects, are incorrect. An exactly solvable model that pinpoints the flaw in the logic was discussed by J. A. Krommes, Phys. Plasmas 4, 655 (1997). However, the basically dimensional result, that sufficiently large shear reduces transport, is upheld by more systematic calculations.

${ }^{24}$ Note that the specific formula used for $\tau_{\mathrm{c}}\left(k_{y}\right)$ obtains only for $\mathcal{S}>1$, where $\widehat{\eta}_{\boldsymbol{k}}$ is diffusive. The definitions (18) are also derived in that limit, although formula (18b) is a valid dimensionless parameter in all regimes.

25 J. A. Krommes and M. Ottaviani, Long-time tails do not necessarily imply self-organized criticality or the breakdown of the standard transport paradigm, submitted to Phys. Plasmas.

${ }^{26}$ R. H. Kraichnan, J. Math. Phys. 2, 124 (1961), erratum: J. Math. Phys. 3, 205 (1962).

27 R. H. Kraichnan, Phys. Fluids 7, 1723 (1964).

28 J. A. Krommes, Phys. Plasmas 4, 1342 (1997).

${ }^{29}$ G. L. Eyink, J. Stat. Phys. 83, 955 (1996).

${ }^{30}$ B. A. Carreras, Bull. Am. Phys. Soc. 43, 1920 (1998).

31 J. A. Krommes, Plasma Phys. Control. Fusion 41, A641 (1999).

32 B. A. Carreras, B. P. van Milligen, M. A. Pedrosa et al., Phys. Plasmas 5, 3632 (1998). 